MIDPI

MOL2NET, International Conference Series on Multidisciplinary Sciences http://sciforum.net/conference/mol2net-03

sciforum

\title{
Plant species with antioxidant capacity
}

<Gemma Martínez> (gemmarlo@mail.ucv.es), <Irene Sarrión> (irene.sarrion@ucv.es), $<$ Gloria Castellano> (gloria.castellano@ucv.es) and <Carmen Fagoaga *> (carmen.fagoaga@ucv.es)

<Universidad Católica de Valencia San Vicente Mártir> *corresponding author

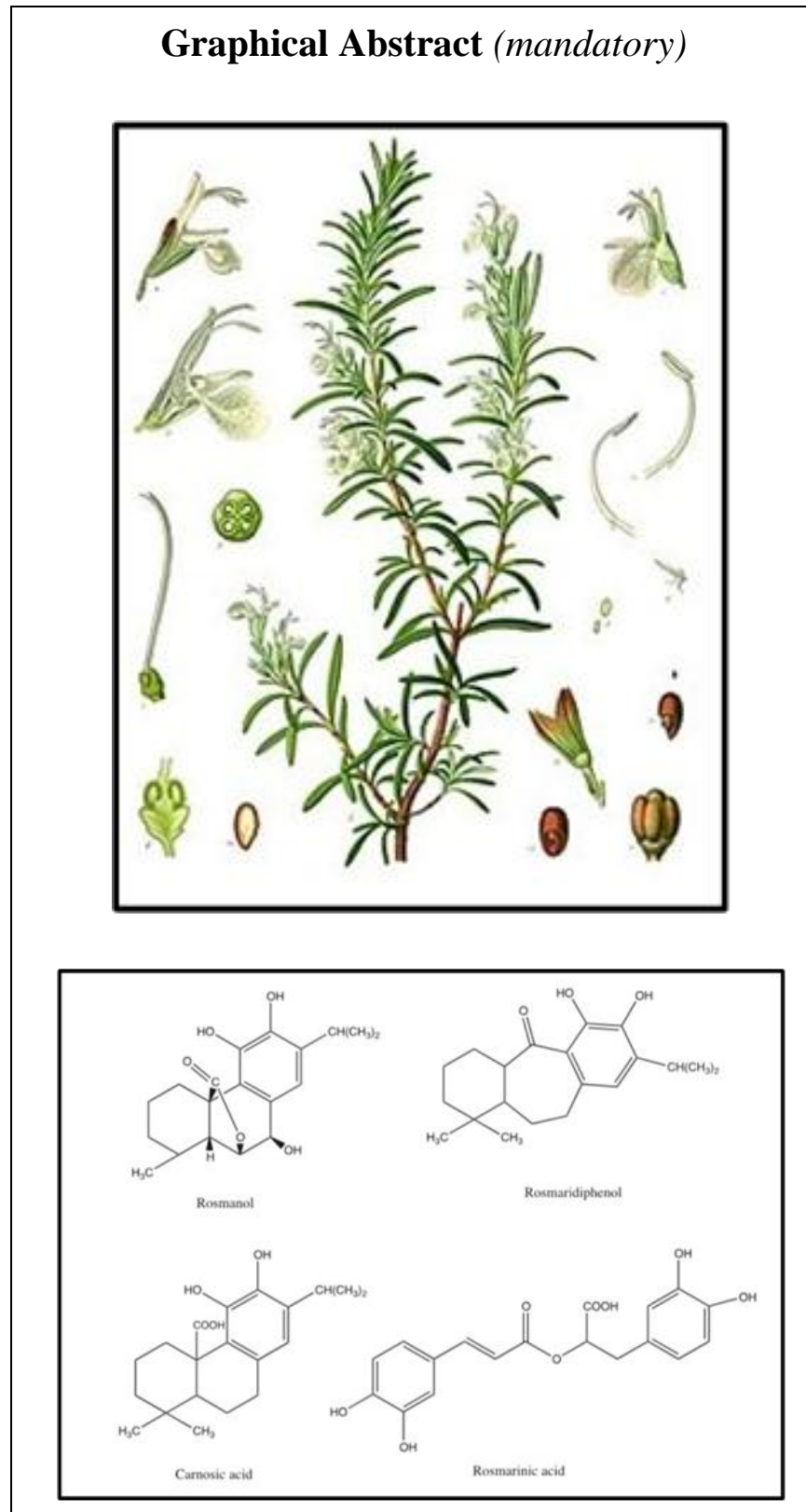

Rosmarinus officinalis L. and chemical structures present in Rosmarinus officinalis (Shahidi \& Ambigaipalan, 2015)

\begin{abstract}
. (mandatory)
Lipid oxidation is the main deterioration reaction during the processing of food, as well as its storage. Both circumstances limit the life of most foods, causing smell and taste to rancidity in them, a process known as oxidative rancidity. In addition, lipid oxidation can damage biological membranes, enzymes and proteins, resulting in the appearance of potentially toxic secondary compounds. To control these oxidative processes, addition of antioxidants is a common strategy against oxidative reactions in the processing or storage of food. The antioxidants commonly used have begun to worry in today's society about the harmful effects they may have on human health.
\end{abstract}

Recently, an industry of natural antioxidants derived from plant species that can replace synthetic antioxidants is emerging. The enrichment of processed foods with vegetable extracts not only solves the problem of oxidation of the food but can also result in an improvement of consumer health. These natural compounds are mainly polyphenols (phenolic acids, flavonoids, anthocyanins, lignins), carotenoids (xanthophylls and carotenes), tocopherols, tocotrienols and some amino acids and peptides. They are widely distributed in species of cereals, aromatics, fruit trees, horticultural crops and oilseeds.

Natural antioxidants present in aromatic plant species such as rosemary (Rosmarinus

officinalis L.) and sage (Salvia officinalis L.) are already being marketed as natural and safe food preservatives, being applied in foods rich in fats such as vegetable oils. 


\section{Introduction}

For hundreds of years antioxidants have been used in food preservation in order to prolong the storage time. They are also used as preservatives of color, flavor, and aroma, as well as to maintain their nutritional value and prolong the life of the product.

Current synthetic antioxidants used in the food industry have undergone different tests to check their safety. However, although they have been used in recent years, there are still doubts about their safety for food use. Studies are required for their possible mutagenic, teratogenic and carcinogenic effects. It has been found that synthetic antioxidants can themselves have toxic properties always at high concentrations. They can also induce DNA damage or have the ability to be cancer initiators (Taghvaei \& Jafari 2015).

Secondary metabolites in plants constitute a huge reservoir of chemical biodiversity. In this work we will focus on explaining those that are involved in antioxidant functions and on introducing those plant species with a high antioxidant capacity that may be useful in the food industry, particularly in processes related to food having a high percentage of lipids, such as fats and oils.

There is also a growing interest in the exogenous addition of these compounds as food enrichment in order to reduce the use of synthetic antioxidants since consumers have expressed a desire for a preference for natural alternatives.

\section{Results (optional)}

Lipid oxidation occurs primarily as a result of oxidative deterioration of polyunsaturated fatty acids (PUFAs) (Reiter, Tan \& Galano, 2014). Oxidation reactions consist of self-oxidations, enzymatic oxidations and photo-oxidations, with auto-oxidation being the most common when it comes to storage and preservation of food (Taghvaei \& Jafari 2015).

Oxidative stability is an important parameter to assess the quality of oils and fats. It refers to the ability to resist oxidative rancidity (or deterioration) during processing and storage periods (Hu \& Jacobsen, 2016). Some of the factors that can influence the oxidative stability of food are the composition of fatty acids, the presence of metals such as iron, antioxidants or photosensitizers.

A diverse range of endogenous antioxidant compounds are found in plant species and numerous publications report the growing evidence that the consumption of a variety of antioxidant compounds present in natural foods of plant origin can reduce the risk of serious disorders in human health due to their antioxidant activities, in addition to other mechanisms.

These vegetable by-products can be classified into several groups according to their chemical structure. These structures vary from simple phenolic acids to more complex structures such as flavonoids, tocopherols, tocotrienols, carotenoids, xanthophylls and various types of amino acids, peptides and proteins.

The addition of these extracts does not cause significant changes in the organoleptic characteristics or in the nutritional value of the food. The plant species used as natural antioxidants come in different 
forms: the plant material, either complete or ground, as an extract, as an oleoresin or as an isolated bioactive compound. Martínez-Tomé et al., (2001) determined that the relative effectiveness of antioxidants depended on the type in which it was applied to a specific substrate (Gutteridge \& Halliwell 2018).

Among plant species with more antioxidant activity, the following stand out: Camellia sinensis (green and black tea with catechin and teaflavin respectively); Peumus boldus Mol. commonly known as boldo, whose major compounds are flavonoids, the best known is boldine, although it also contains reticulins, kaempferitrins and quercetins; Sesamum indicum $L$. whose seeds are rich in phytochemicals known as lignans, as well as vitamins, phytosterols and polyunsaturated fatty acids; Ocimum basilicum L. basil extract has rosmarinic acid which is the most abundant phenolic compound; Olea europaea (the olive tree), whose leaves contain five groups of phenolic compounds: oleuropeosides, catechins, flavones, flavonols and phenols; Glycyrrhiza glabra, (licorice) which containing triterpenoid saponins and flavonoids as the main phenolic compounds and Zingiber officinale Rosc. where volatile compounds present as gingerols, shogaols, paradoxes and zingerone have antioxidant activity by elimination of free radicals.

To conclude, add that the use of rosemary (Rosmarinus officinalis L.) and sage (Salvia officinalis L.) extracts are already formally accepted in the European regulations as a new food additive and assigned with its number E-392 (Tohma \& Turan, 2015).

\section{Conclusions (optional)}

1. Lipid oxidation has been identified as one of the processes that most deteriorates vegetable oils, affecting both the organoleptic characteristics and the nutritional quality of the food.

2. Numerous plant species contain electron donors that can prevent free radical formation reactions and slow down or inhibit oxidative processes.

3. Plant natural extracts are safer than synthetic antioxidants and can bring healthy benefits to the consumer, as well as reduce the risk of disease.

4. Rosmarinus officinalis L. and Salvia officinalis L. contain numerous active compounds, of which carnosic acid, carnosol and rosmarinic acid have been identified as the majority. These compounds are also present in other plant species, such as Ocimum basilicum $\mathrm{L}$

5. The following plant species: Camellia sinensis, Peumus boldus Mol., Sesamum indicum L., Olea europaea, Glycyrrhiza glabra and Zingiber officinale Rosc. contain antioxidant compounds with names referred to the own species (e.g. teaflavin, boldine, sesamol, etc...) because they have not been found in other plant species at the moment.

6. Extracts of plant species are already being used as natural antioxidants in the protection of taste, rancidity and storage of oils of plant origin.

7. There are more than 70 plant species with antioxidant capacity mentioned in this review, some 
should be studied more in depth by the scientific community in the future, due to the importance they can acquire in the coming years as fortified foods or as food additives with preservative function.

8. These data support the replacement of the synthetic antioxidant by such natural spice extracts that because of their contribution of characteristic colors and flavors would prompt the use of these spices in the design of new functional foods.

\section{References (mandatory)}

Gutteridge, J. M. C. \& Halliwell, B. (2018). Oxidative Stress, redox stress or redox success? Biochemical and Biophysical Research Communications, 502 (2), 183-186. doi:10.1016/j.bbrc.2018.05.045

Hu, M \& Jacobsen Ch. (2016). Mini-review Oxidative Stability and Shelf Life of Foods Containing Oils and Fats, San Diego, CA, USA, Elsevier

Martínez-Tomé, M., Jiménez, A.M., Ruggieri, S., Frega, N., Strabbioli, R. and Murcia M.A. (2001) Antioxidant Properties of Mediterranean Spices Compared with Common Food Additives. Journal of Food Protection, 64 (9), 1412-1419. doi:10.4315/0362-028X-64.9.1412

Reiter, R., Tan, D., \& Galano, A. (2014). Melatonin reduces lipid peroxidation and membrane viscosity. Frontiers in physiology 377 (5)1-4. doi: 10.3389/ fphys.2014.00377

Shahidi, F. \& Ambigaipalan, P. (2015). Phenolics and polyphenolics in foods, beverages and spices: Antioxidant activity and health effects- A review. Journal of Functional Foods, 18, 820-897.

Taghvaei, M., \& Jafari, S. M. (2015). Application and stability of natural antioxidants in edible oils in order to substitute synthetic additives. Association of Food Scientists \& Technologists, 52, 1272-1282.

Tohma, S., \& Turan, S. (2015). Rosemary plant (Rosmarinus officinalis L.), solvent extract and essential oil can be used to extend the usage life of hazelnut oil during deep frying. European Journal of Lipid Science and Technology 117 (12), 1978-1990. doi:10.1002/ejlt.201400382 